\title{
La Investigación-acción participativa en la metodología docente universitaria. Una experiencia de innovación desde la perspectiva de género ${ }^{1}$
}

\section{Participatory action research in the university teaching methodology. An innovation experience \\ from a gender perspective}

Irene Martínez Martín

Universidad Complutense de Madrid, España

imarti02@ucm.es

Teresa Rabazas Romero

Universidad Complutense de Madrid, España

rabarom@ucm.es

Carlos Sanz Simón

Universidad Complutense de Madrid, España csa02@ucm.es

Ainhoa Resa Ocio

Universidad Complutense de Madrid, España aresa@ucm.es

\begin{abstract}
Resumen: Las metodologías participativas en el ámbito docente universitario tienen especial relevancia considerando las últimas reformas legislativas en torno a los Espacios Europeos de Educación Superior (EEES). En este artículo se analizan las dinámicas de participación del alumnado y el profesorado universitario desde un enfoque de género dentro del marco de un proyecto de innovación docen-
\end{abstract}

${ }^{1}$ El presente artículo se ha llevado a cabo gracias a la concesión de un contrato predoctoral de personal investigador en formación cofinanciado por la Universidad Complutense de Madrid y el Banco Santander (Convocatoria 2017, CT17/17-CT18/17). 
te, aplicado en diversas asignaturas y facultades de la Universidad Complutense de Madrid en el curso 2019-20. La metodología de esta iniciativa parte de un proceso de investigación acción participativa (IAP), que se ha desarrollado en tres fases: observación, autorreflexiones y propuestas de transformación. Tras el análisis de las dinámicas de clase, se ha evidenciado que existen desigualdades en la participación del alumnado en función del género, y a partir de éstas se proponen estrategias de mejora que promuevan una formación del profesorado universitario en igualdad.

Palabras clave: formación del profesorado, igualdad de género, participación, investigación acción participativa

Abstract: Participatory methodologies in the university teaching field have special relevance considering the latest legislative reforms around the European Higher Education Spaces (EHEA). This article analyzes the dynamics of participation of students and university teachers from a gender perspective within the framework of a teaching innovation project, applied in various subjects and faculties of the Complutense University of Madrid (2019-20). The methodology of this initiative is based on a participatory action research (IAP) process, which has been developed in three phases: observation, self-reflections and transformation proposals. After the analysis of class dynamics, it has been shown that there are inequalities in the participation of students according to gender and from these, improvement strategies are proposed that promote a training of university teachers in equality.

Keywords: teacher training, gender equality, participation, participatory action research. 


\section{INTRODUCCIÓN}

Bajo los principios de la educación crítica y contrahegemónica (Freire, 1969; Mclaren, 1997; Giroux, 2019), la participación activa queda señalada como un elemento esencial para lograr de la educación un acto de empoderamiento individual y colectivo (Álvarez, 2001). No podemos pensar la participación y la educación sin tener en cuenta una perspectiva de género derivada de las pedagogías feministas que cuestionan las dinámicas de agencia, la ocupación de espacios (públicos y simbólicos) y el uso que de ellos se hace, de manera diferenciada en función de la pertenencia, o no, a los grupos hegemónicos (Martínez, 2017).

Este contexto epistemológico queda enmarcado en una investigación en curso impulsada por la unidad de igualdad de la Universidad Complutense de Madrid (proyecto SUPERA, Supporting the Promotion of Equality in Research and Academia, EU $\mathrm{H} 2020)^{2}$, que visibiliza la realidad de desigualdad en los diversos espacios universitarios, atendiendo a la igualdad de género en la carrera universitaria, a los liderazgos y a la toma de decisiones en investigación, docencia y vida universitaria, y estereotipos, sesgos de género, acoso sexual y sexista. En la fase inicial de diagnóstico, en este proyecto se ha puesto de manifiesto una serie de carencias, entre ellas, la falta de metodologías docentes que favorezcan dinámicas de género, inclusivas y participativas en la cotidianidad del aula, así como la formación del profesorado universitario y la difusión de buenas prácticas que se realizan en esta materia y que, en muchas ocasiones, quedan relegadas al espacio privado de cada docente.

Como parte de las acciones impulsadas en este marco, en la Facultad de la Educación de la UCM se desarrolla un proyecto de innovación docente durante el curso 201920 titulado «Metodologías participativas e innovadoras en la docencia universitaria desde un enfoque de género». Surge ante el cuestionamiento de las carencias metodológicas indicadas en los procesos de enseñanza y aprendizaje a la hora de favorecer dinámicas participativas con enfoque de género en la cotidianidad del aula. El proyecto tiene como finalidad mejorar la formación del profesorado universitario impulsando buenas prácticas vinculadas a esta materia.

En este contexto se justifica la necesidad de analizar nuestras dinámicas de aula poniendo el acento en la participación activa y consciente desde una perspectiva de gé-

${ }^{2}$ El proyecto SUPERA está liderado por la profesora María Bustelo, y en él se integra una red de nodos formada por una representante de cada facultad. En la Facultad de Educación, el equipo está integrado por los participantes del proyecto de innovación docente presentado en este artículo. 
nero. A su vez, este proyecto pretende ser coherente con los principios de la enseñanza universitaria basada en el plan Bolonia, donde el alumnado es agente activo de su aprendizaje y el profesorado tiene entre sus competencias alentar prácticas de enseñanza dinámicas, participativas, inclusivas y creativas.

El objetivo principal de este proyecto de innovación consiste en analizar y visibilizar las dinámicas de participación que, desde un enfoque de género, se dan en las aulas universitarias, poniendo en cuestión los componentes de desigualdad que intervienen en la cotidianidad académica.

En el desarrollo del proyecto se plantea una metodología cualitativa basada en los principios de la investigación acción participativa. Se usan técnicas como la observación participante, el autoanálisis y los grupos de debate para la toma de conciencia crítica. Dicho proceso se estructura en tres fases: 1) recogida de datos y observaciones participantes; 2) dinámica de autorreflexión y análisis de datos colaborativo; y 3) observación y propuestas de mejora.

Respetando el objetivo planteado, se busca dar respuesta a una serie de preguntas al alumnado, cuestionando si existe una participación libre, igualitaria y consciente en las aulas universitarias, si afecta la socialización diferenciada de género en nuestras dinámicas de participación académicas, si hay espacios simbólicos en las aulas ocupados por hombres y silenciados por mujeres en relación con la participación, etc. Respecto al profesorado, se pretende conocer si favorece una participación libre de estereotipos de género, cómo actúa cuando los alumnos ocupan los espacios de participación sin dar posibilidad de diálogos constructivos y qué conductas docentes contribuyen a mantener el statu quo y se pretenden transformar.

Entre las propuestas finales se propone la construcción de un conocimiento colectivo desde los principios de la pedagogía feminista y en coherencia con el proyecto de investigación SUPERA para generar pautas de acción con el fin de que mejoren la labor docente universitaria en materia de metodología participativa con enfoque de género.

\section{PARTICIPACIÓN UNIVERSITARIA DESDE UNA PERSPECTIVA DE GÉNERO}

Este artículo se contextualiza en los principios de la pedagogía feminista y crítica. Giroux (2019) señala la importancia de entender la educación como un proceso de empoderamiento y transformación. Así, hace saber que la educación no es neutra. Puede estar al servicio de las clases hegemónicas y servir para la reproducción de desigualdades o, por el contrario, puede ser una práctica de libertades, emancipación y crítica. Conseguir que la educación sea un acto consciente de transformación, de construcción 
de ciudadanías justas y en equidad, de toma de conciencia de nuestras realidades, del lugar que ocupamos en la relación opresión-privilegio y educar más allá de la mera transmisión y memorización de contenidos (Freire, 1969) es el camino a seguir por las pedagogías críticas y de liberación.

En esta misma línea, las pedagogías feministas se cuestionan la construcción y transmisión de las desigualdades en los espacios de poder, en la toma de decisiones, los techos de cristal, las brechas de género y las diversas violencias machistas (simbólicas o físicas), y la responsabilidad que la educación tiene en ello. Siguiendo a Martínez (2018) o Sánchez (2019), construir una educación feminista pasa por cuestionar el complejo mundo de privilegios e intersecciones (Crenshaw, 2000) de opresiones (de género, raza, clase, identidad sexual, económica, de funcionalidad...), así como por incluir las diversidades, las igualdades y la justicia social como elementos básicos de la educación. Pensar y hacer pedagogía feminista es tomar conciencia del lugar que ocupamos, pero también desmontar, repensar y reconstruir nuestras prácticas sociales (individuales y colectivas), estereotipos de género, desigualdades y violencias machistas en favor de un mundo diverso, empoderante, emancipador y comunitario, rompiendo con las imposiciones de la normatividad individualista y competitiva.

La pedagogía feminista es participativa (Hipólito, Martínez y Fernández, 2019). Hablar de participación feminista o con enfoque de género (Lagarde, 2000) es prestar atención a la participación política y activista, pero también al empoderamiento individual teniendo en cuenta la mirada de las mujeres, su presencia, así como las dificultades e invisibilidades. Por tanto, cuando definimos la participación activa podemos atender a diferentes enfoques y perspectivas, ya sea desde un análisis individual o desde análisis colectivos, políticos y activistas. La participación es más que mera información, no se limita a una colaboración puntual o a un encuentro en un espacio público, o el apoyo puntual a alguna acción. Participar, desde el ámbito social, es un acto significativo, consciente, gradual, con objetivos inmediatos, a medio y largo plazo, con fines estratégicos. Es decir, intervienen dinámicas individuales y grupales donde aparece el poder de decisión y las posibilidades de control, el trabajo colectivo, la organización y la planificación, el compromiso y la responsabilidad por el bien común y los fines compartidos.

Educar en la participación así definida es un reto de nuestros espacios académicos. Las autoras anteriores señalan que a participar se aprende participando, cediendo espacios de poder y abriendo canales diversos para el diálogo y el intercambio, ensayando fórmulas diversas para la toma de decisiones y la resolución de conflictos, transformando metodologías y dinámicas verticales y autoritarias, educando en valores cooperativos y colectivos, desterrando las prácticas sociales y educativas tradicionales, competitivas e individualistas, entre otras. 
Tonucci (2009) se refiere a la participación como un derecho que debe ser contemplado por la educación. Se pregunta si se puede enseñar la participación, si se puede enseñar la democracia y cómo se debe educar en la participación libre. Desde este artículo añadimos: ¿se puede participar sin etiquetas y desigualdades de género?

Ante esto, nos encontramos con una desigualdad de género institucionalizada y presente en las esferas universitarias — reflejo de la sociedad patriarcal— que se hace patente en brechas de género en las diferentes carreras universitarias, en los techos de cristal, la masculinización o feminización de ciertas profesiones o tareas y en la reproducción de estereotipos sexistas y sus violencias. Un ejemplo claro lo encontramos en la feminización de la Facultad de Educación de la UCM, donde, a pesar de haber mayoría de mujeres, encontramos sesgos de género en temas como la participación pública, el reparto de tareas de liderazgo o la subida en la escala académica, por citar algunos de los elementos relacionados con el citado proyecto SUPERA. La misma situación queda reflejada en otras disciplinas científicas participantes en este proyecto y tradicionalmente masculinizadas como ciencias físicas o químicas, donde esta desigual participación es igualmente palpable.

El modelo social en que está instalada nuestra sociedad es machista y desigual. Los feminismos y las mujeres han luchado y reivindicado la ocupación de espacios tradicionalmente masculinos, han tomado la voz pública y han cuestionado las estructuras de poder tradicionales que relegan a las mujeres a los espacios privados - invisibilizados y no valorados - . Las mujeres han luchado por incorporarse a la educación, al empleo, a vivir libres y con seguridad, a la igualdad salarial, a la participación política..., ocupando espacios que han sido tradicionalmente negados a las mujeres. Hablamos de alcanzar la igualdad real y de derechos y no solo la igualdad formal (Lagarde, 2014). Paro ello, tampoco podemos olvidar el gran reto que supone el posicionamiento de las masculinidades dominantes que se resisten a abandonar sus privilegios y transformar esta dualidad de vida privada dedicada a los cuidados frente a la vida pública y productiva.

Por otro lado, la organización social sigue respondiendo a valores machistas donde se reproducen estructuras de desigualdad, individualismo y competitividad. Desde una perspectiva feminista se busca subvertir este orden de opresión y privilegios poniendo en el centro un proyecto colectivo de transformación. Esta perspectiva subraya la necesidad de un compromiso colectivo para actuar conjuntamente, denunciar las desigualdades y construir nuevos canales de participación y educación no sexistas (Subirats, 2017).

En el contexto universitario, se requiere un alto compromiso y toma de conciencia crítica del lugar que el profesorado ocupa en la reproducción de dichas desigualdades bajo la finalidad compartida de cambiar el modelo social de participación. En este camino de transformación, la educación crítica y la pedagogía feminista sientan las claves 
para (Martínez, 2016) proponer que la participación de las mujeres sea algo más que lograr el derecho al voto. Ampliar el derecho a la participación supone construir desde lo colectivo, buscar mecanismos de acción positiva y transformadores para subvertir el orden machista dominante. Caminar hacia una sociedad en la que hombres y mujeres compartamos responsabilidades y logros desde la equidad y la justicia social (Martínez y Escapa, 2008).

Analizar la participación desde una perspectiva de género supone también atender a las brechas sexistas en dicha participación. Entre otras muchas cuestiones cabe preguntarse si las mujeres ocupan puestos de gobernanza y públicos, si en el escenario político las mujeres participan en posiciones de igualdad, si están excluidas de los espacios de poder y participación, si se respeta su derecho a participar, o bien, si los espacios de poder y participación son ideados por hombres y para hombres. Cualquier proceso participativo que tenga en cuenta una perspectiva feminista debe trabajar por desmontar los mitos que frenan la participación (Hipólito, Martínez y Fernández, 2019): las mujeres no son capaces de liderar, el poder es cosa de hombres, tienes que elegir entre tu trabajo y la maternidad, etc.

Es necesario transformar esa idea de poder tradicional ligada a los valores masculinos-patriarcales para acercarnos a formas de poder colectivas, feministas y transformadoras de construcción de ciudadanías. Para ello, las pedagogías feministas y la educación crítica proponen transformar, entre otros aspectos, las dinámicas de participación tradicionales desde bases coeducativas, en equidad, con justicia social y respetuosas con las diversidades.

\section{DISEÑO METODOLÓGICO Y DESCRIPCIÓN DEL PROCESO DE IAP}

Se propone un enfoque cualitativo dentro de un paradigma de investigación crítico y emancipador (McLaren, 1997; Habermas, 2000) y desde una perspectiva dialéctica (Ibáñez, 1990). Se busca el cuestionamiento de una realidad concreta para su transformación y mejora desde la práctica, así como el impulso de la participación como estrategia investigadora.

\section{Objetivos del proceso de IAP}

En el caso aquí presentado, dicho cuestionamiento se plantea desde los seis objetivos principales del proyecto de innovación docente: 
- Analizar las dinámicas de participación (visibles y no visibles) del alumnado y el papel que juega el profesorado en éstas.

- Visibilizar dichas dinámicas de participación desde un enfoque de género en las aulas universitarias (quiénes participan, cómo participan y quiénes no participan).

- Poner en cuestión los componentes de desigualdad de género que intervienen en la cotidianidad de las aulas y que frenan la participación activa de las mujeres y/o impulsan la participación activa de los hombres.

- Tomar conciencia crítica individual y colectiva acerca de los componentes que favorecen o dificultan la participación desde un enfoque de género.

- Transformar las dinámicas de participación en el aula desde un enfoque de género e inclusión, atendiendo a los roles del profesorado y el alumnado, la metodología de enseñanza y aprendizaje, los lenguajes y comunicación, el uso de espacios simbólicos y la evaluación.

- Generar espacios de formación entre alumnado y profesorado proponiendo acciones metodológicas que tengan en cuenta los principios de inclusión, participación e igualdad.

La consecución de esta finalidad parte del método de investigación-acción participativa, cuyos exponentes fundamentales son Fals Borda (1990), Foote (1991), Kemmis (1992), Villasnte (2002), Noffke \& Somekh (2009) y Caballero, Martín y Villasante (2019). También experiencias de aplicación de la IAP en estudios de género desde el ámbito de las ciencias sociales (Orefice, 2014; Breckmans, Velasco \& Loots, 2016), donde resultan destacables los casos de Berge \& Ve (2000) y Naples (2003), los cuales aplican este método en el campo del feminismo. En España hay que destacar los estudios y prácticas docentes de Oliveira \& Waldenez (2010), Barba, Martín \& Barba (2015), Barba, Barba \& Martínez (2016) y Barba \& Sanz (2019) en el campo de la educación, y el de Barba (2019) en relación con la formación de maestras.

\section{Muestra y participantes}

Este proceso tiene lugar en distintas materias impartidas en las facultades de Educación de la Universidad Complutense de Madrid y de Valladolid. En el caso de la primera, se lleva a cabo en materias comunes a todo el alumnado del centro, como Historia y corrientes internacionales de la Educación, Didáctica e innovación curricular, Organización y gestión de instituciones educativas y de otras como Historia del pensamiento pedagógico — perteneciente al Grado en Pedagogía - Desarrollo comunitario - im- 
partida en el Grado en Educación Social — o las materias de La investigación histórica y comparada en Educación e Internacionalización y reformas educativas. Perspectivas globales y de género - pertenecientes al Máster Universitario en Investigación en Educación - de la UCM. En el caso de la segunda, en la materia Tecnologías de la Información y la Comunicación Aplicadas a la Educación del grado en Magisterio de Educación Primaria. Por otra parte, también se cuenta con otros centros colaboradores, como las facultades de Enfermería, Físicas, Políticas y Químicas de la Universidad Complutense, con la finalidad de establecer una comparación entre las facultades de ciencias sociales y las de ciencias experimentales.

Con esta participación de asignaturas, la muestra es variable debido a las agrupaciones de prácticas propias de cada asignatura. Se hace una estimación de la participación de estudiantes, siendo un total de 400 . Entre estos, el porcentaje femenino de estudiantes es de un $80 \%$, siendo una muestra feminizada por las características propias de los estudios y asignaturas seleccionadas.

Los recursos humanos de la investigación, además del estudiantado, han sido el profesorado participante en el proyecto de innovación (13), siendo 9 mujeres y 4 hombres.

\section{Proceso y enfoque de análisis}

Dentro de este contexto académico, el presente proyecto parte de una distribución metodológica en torno a tres fases principales, que se secuencian del siguiente modo (y cuyo organigrama puede consultarse en el Anexo 1):

1. Recogida de datos y observaciones. Durante el primer mes de las asignaturas se realiza un registro de observación participante por parte del profesorado y del equipo del proyecto. Esta observación tiene lugar en dos sesiones escogidas aleatoriamente en cada materia, con la intención de registrar los patrones de participación desde el enfoque de género, teniendo en cuenta una serie de indicadores que permitan que dicha observación sea comparable entre casos (ver Anexo 2), a saber:

a. Nivel de participación general de la clase: intervenciones en voz alta, presencia de chicos y chicas, clima de clase y palabras solicitadas voluntariamente.

b. Estrategias o prácticas del profesorado que favorecen o dificultan la participación: preguntas a la clase, turno de palabra aleatorio, uso de dinámicas con pequeños grupos, apertura de debates generales, la propia gestión de las intervenciones del alumnado o el uso de nuevas tecnologías para la participación en el aula, entre otras. 
c. Número de personas que participan.

d. Tipo de intervenciones y su pertinencia en el contenido y desarrollo del debate, codificando las intervenciones en función de un conjunto de marcadores que aluden al tipo de intervención, como los siguientes casos:

i. Introduce un argumento o expone una idea nueva $v s$. repite una idea que ya ha explicado él/ella anteriormente.

ii. Recoge las intervenciones de los demás y se apoya en ellas para contribuir al debate grupal $v s$. ignora las aportaciones del resto y habla sobre sus propios intereses.

iii. Utiliza lenguaje y componentes asertivos $v s$. lenguaje y componentes agresivos o pasivos.

2. Sesión o taller formativo y autoanálisis (Ioé, 2010) del alumnado de las dinámicas de participación durante las clases. Esta autoevaluación de las propias intervenciones del alumnado se realiza en un registro previo realizado de forma individual (ver Anexo 3) y responde a las siguientes cuestiones: ¿cuántas veces participo? ¿cómo es mi participación?, ¿por qué no participo?, ¿qué me lo impide?, ¿quién participa más?, ¿cómo son mis intervenciones?, ¿y las intervenciones ajenas?, ¿favorece el profesorado la participación?, ¿cuándo me siento más interpelado/a a intervenir?, ¿detecto diferencias de género en la participación?

Tras esta primera parte individual, se redistribuye a la clase en pequeños grupos, en los cuales comparten las reflexiones propias en torno a su participación. Para ello se realiza la dinámica grupal de la «flor de loto» para debatir en torno a los problemas de participación detectados y en la propuesta de soluciones por parte del alumnado ${ }^{3}$. Esta técnica permite una sesión abierta de debate y reflexión conjunta entre profesorado y alumnado en la que se ponen en común los análisis de participación, los problemas detectados y sus posibles soluciones. Finalmente, cabe destacar que en esta sesión se incorporan integrantes del equipo del proyecto para dinamizar las sesiones, atendiendo a cuestiones como: ¿es la participación algo importante en el aprendizaje?, ¿qué me hace participar y que no?, ¿la socialización diferenciada de género en la construcción de masculinidades y de feminidades interviene (obstaculizando o favoreciendo) la participación?, ¿cambian las interacciones dentro del aula si se toma conciencia de las dinámicas de participación en función del género?

${ }^{3}$ La técnica de la «flor de loto» fue creada por Yasuo Matsamura, presidente de Clover Management Research, y consiste en la realización de asociaciones mentales a través de conceptos de forma gráfica, con el fin de desarrollar ideas creativas partiendo de una temática o problema principal. 
3. Observación participante, autoevaluación del profesorado y propuestas de mejora.

La primera técnica de esta fase se realiza en dos sesiones aleatorias de cada materia. En ella se registran los patrones de participación desde un enfoque de género, utilizando para ello los indicadores de la primera fase.

En segundo lugar, se realiza una autoevaluación del personal docente de las diversas materias impartidas, en la cual se recogen sus propias percepciones sobre la participación en las aulas, así como las iniciativas metodológicas y didácticas que han tenido lugar durante el progreso de las materias para favorecer - $\mathrm{O}$ nola participación libre y equitativa. El formulario de autoevaluación del profesorado puede consultarse en el Anexo 4.

En último lugar, y como cierre del proceso, se concluye con la transferencia de los resultados. Para ello se realiza una jornada formativa para el profesorado y el alumnado de la Universidad Complutense de Madrid, donde se ponen en común las líneas reflexivas y los principales resultados obtenidos durante todo el proceso. Para ello se utiliza el formato «coffee-table», para crear un espacio abierto y dinámico que permita la creatividad y accesibilidad para participar e intervenir libremente.

El proceso de análisis realizado una vez desarrolladas las fases de la IAP descrita ha sido una parte más de la IAP. A partir de las técnicas de observación y las técnicas grupales de investigación (Ioé, 2010), se ha usado el debate grupal y el autoanálisis de los propios procesos de participación como elementos que han permitido poner en común los resultados, cuestionarlos, explicarlos y construir nuevos elementos del discurso. Todo ello como parte fundamental del proceso de IAP.

\section{PRINCIPALES ANÁLISIS DEL PROCESO INVESTIGADOR: ¿SON EQUITATIVOS LOS PROCESOS PARTICIPATIVOS EN LA DOCENCIA UNIVERSITARIA?}

A continuación se expone el análisis de los datos obtenidos a partir de la observación participante por parte del profesorado y del equipo del proyecto, así como de los talleres formativos y de autoanálisis de participación del alumnado durante las clases. Tal y como se ha explicado anteriormente, estas sesiones se desarrollan en torno a una serie de preguntas sobre la participación individual de cada persona, sus características, 
los factores que la favorecen y dificultan, y su percepción sobre la relación entre la participación y el género.

En primer lugar, a partir de los registros de observación participante del profesorado y equipo del proyecto, se puede ofrecer un primer acercamiento a la participación general del alumnado. A lo largo de las clases, las y los docentes han apreciado que la participación es mayor en las sesiones prácticas, de dinámicas y de debate. Por norma, han identificado que quienes más participan son, en proporción, los chicos y las chicas con perfil de liderazgo. Los alumnos, a pesar de ser menos, tienden a intervenir en todas las clases y a ocupar roles de líderes en las exposiciones o a través de bromas y comentarios. Las alumnas, por el contrario, cuando participan suele ser para argumentar fundamentando sobre la temática de la asignatura. Las voces más reconocidas y de liderazgo no suelen variar y la participación es desigual, sin embargo, las intervenciones se producen con respeto y los conflictos se resuelven a través del debate.

Por otra parte, los datos recogidos a través del autoanálisis del alumnado permiten ahondar en su participación en las clases. En el conjunto de los grupos analizados, existen diferencias entre la participación manifestada por las diferentes personas: algunas se consideran activas y participativas, otras califican su participación como media y, por último, existen aquellas que no intervienen nunca o casi nunca en las clases. Se dan incluso casos de personas que no llegan a participar en ninguna ocasión a lo largo del cuatrimestre. A pesar de que todas se identifiquen en uno de estos grupos, se aprecia una participación diferenciada en función del género. Los hombres tienden a situarse en el grupo de las personas con una alta participación junto con las mujeres líderes, mientras que el conjunto de aquellas que no intervienen nunca o casi nunca está conformado mayoritariamente por chicas. Asimismo, es mayoritario el grupo de personas que no participan de manera activa y libre, pero se ha podido comprobar cómo la participación aumenta conforme avanzan las sesiones y el alumnado siente una mayor confianza con sus compañeras y compañeros.

En cuanto a qué cuestiones les impiden participar, existen diferencias significativas entre las respuestas expresadas por las chicas y los chicos. Por una parte, las alumnas, al explicar los inhibidores de su participación, aluden con gran frecuencia a factores disposicionales, esto es, competencias anteriormente desarrolladas, características de la personalidad y motivaciones. Las mujeres, en su mayoría, se refieren a la inseguridad y a aspectos ligados con la autoestima como son la vergüenza, la timidez, la falta de confianza al compararse con otras compañeras o el miedo al rechazo. Existe entre ellas un sentimiento generalizado de inferioridad y una minusvaloración de sus capacidades que entorpece su implicación activa con el conjunto del grupo. Igualmente, argumentan cómo los conocimientos previos suponen una limitación para ellas: la falta de conoci- 
miento sobre la temática a tratar y la dificultad al hablar en público o al expresar ideas son elementos que reducen su participación. Las alumnas no revelan su opinión en el caso de que no cuenten con conocimientos previos sobre el objeto de debate o no tengan la capacidad de elaborar un discurso sólido al respecto. El clima de clase y el interés personal que desarrollen hacia la asignatura también son claves en la participación de las mujeres. De la misma manera, hablan de factores situacionales —el profesorado, el tamaño del grupo y la metodología — al manifestar una menor participación en grupos grandes y determinadas dinámicas establecidas por las y los docentes, pero esto se relaciona estrechamente con el miedo a hablar en público y la inseguridad al confrontarse con sus iguales. En último lugar, y en relación con los impedimentos en la participación de las mujeres, resulta llamativo el hecho de que las alumnas, en numerosas ocasiones, expresan que su participación se ve condicionada por aspectos emocionales (entre las respuestas destacan «según el día que tenga», «depende de si tengo un mal día», etc.).

Por el contrario, las limitaciones en la participación de los hombres se deben, en su mayoría, a factores externos a ellos. Aluden a motivos más racionales y relacionados con aspectos académicos. El interés que les suscite el tema, la metodología y el profesorado son determinantes para su participación en las clases. En lo relativo a los factores disposicionales, se aprecia una gran diferencia con respecto a las alumnas: no expresan temor a hablar en público, temor a las críticas, al juicio de sus compañeras y compañeros, etc., y en tan solo algunos casos expresan que el desconocimiento sobre el tema suponga una barrera. Además, no muestran inseguridad o preocupación ante el juicio colectivo en ningún caso. En lo relativo a este grupo, es especialmente sorprendente el número de chicos que refleja que no existen impedimentos de ningún tipo que alteren y frenen su participación. Con esto, vemos que las alumnas cuentan con más elementos que dificultan su participación al tiempo que tienden a tener mayores inseguridades y miedos que los alumnos - quienes se refieren a cuestiones externas, en el caso de que éstas impidan su participación-.

Del mismo modo que encontramos factores que impiden la participación, identificamos también elementos que la favorecen durante las clases. De nuevo, se pueden ver diferencias en aquellas cuestiones que fomentan la intervención de las chicas y los chicos. En el caso de las alumnas, predominan cuestiones como que son los debates en pequeños grupos, un buen clima, contar con la confianza de sus compañeras o un espacio libre de juicios. Al igual que se puede ver a través de los inhibidores de la participación, en el caso de las chicas, puede mejorar su participación contar con un espacio seguro en el que expresar sus opiniones. También, realizar actividades prácticas, el uso de materiales variados (vídeos, dinámicas, actividades) y prepararse previamente para la exposición oral de sus ideas mejora la confianza de las chicas con sus argumentos y el 
grupo. Sin embargo, en el caso de los alumnos, recalcan su interés personal por la asignatura, por el profesorado y por dar una visión crítica al debate como elementos clave para su participación. Igualmente, contar con propuestas de trabajos motivadores o que supongan un reto y las actividades innovadoras destacan entre los chicos. Una vez más, aluden a elementos externos y a la intervención y las propuestas del profesorado más que a cuestiones individuales o de la personalidad. En algunas ocasiones sí se aprecian similitudes con el grupo de las chicas cuando se refieren a sentirse libres de juicios en las clases.

A partir del análisis de la información realizado, se detecta una desigualdad en la participación en función del género, así como factores diferenciados que afectan de manera positiva y negativa a la misma. Sin embargo, a través de las sesiones de autoanálisis se recabó también información sobre la percepción del alumnado. Así, se ha visto cómo, si bien es cierto que el alumnado es consciente de las diferencias individuales en la participación, no considera, en la mayoría de los casos, que existan distinciones por cuestión de género. Reiteran que las diferencias que se dan son condicionadas por aspectos de la personalidad y sólo en algunos casos son conscientes de las diferencias que existen entre las alumnas y los alumnos y que generan desigualdades y jerarquías en la ocupación simbólica de los espacios de clase.

\section{CONCLUSIONES Y ESTRATEGIAS DE MEJORA PARA LA TRANSFORMACIÓN DE METODOLOGÍAS DOCENTES}

Tras el análisis de los resultados se puede afirmar que en las aulas universitarias se evidencian algunos factores que provocan desigualdades en las dinámicas de participación de los estudiantes desde un enfoque de género. En general, se aprecia una tendencia a que los alumnos ejerzan un mayor liderazgo respecto a las alumnas, siendo el porcentaje de varones muy inferior si lo comparamos con el número de mujeres matriculadas en las carreras de educación. Las explicaciones que argumentan los chicos respecto a su grado de participación también son diferentes a los de las chicas. Los alumnos que no participan esgrimen argumentos relacionados con factores externos, como puede ser la falta de conocimiento de la temática, el escaso de interés por la asignatura, el profesorado, la metodología... Sin embargo, las alumnas exponen que la ausencia de las intervenciones se debe a factores internos, vinculados a cuestiones emocionales o de personalidad: timidez, inseguridad, temor a las críticas, etc.

En relación con la labor docente del profesorado se constata que la propia metodología del profesorado favorece o condiciona la participación del alumnado, ya que, 
como se ha evidenciado, las alumnas pueden inhibir su iniciativa con las dinámicas docentes. En este sentido, es tarea del profesorado estimular la participación de las estudiantes, tomar conciencia sobre las propias metodologías didácticas, evaluar el propio trabajo y buscar las estrategias para su mejora.

A partir de la identificación de estas desigualdades, y siguiendo con la dinámica de la IAP, se ha propuesto una serie de estrategias de mejora para la transformación de metodologías docentes desde los principios de la pedagogía feminista y crítica. Es decir, estas estrategias son consensuadas entre profesorado y estudiantado, nacen de la observación, el debate, el diálogo, el cuestionamiento crítico y la construcción colectiva de saberes y prácticas. Cabe destacar: a) fomentar un espacio seguro, un buen clima de clase y sin que repercuta negativamente en la calificación; b) promover una mayor dinamización de los grupos de trabajo, alternando su composición y el tamaño, proponiendo actividades prácticas, debates, vídeos, etc.; c) usar diversas vías de comunicación para que la participación oral no sea la única forma de tener participación activa (participación online, chat, vídeo, tutorías, propuestas de materiales...); d) incluir dinámicas de trabajo donde la participación sea rotatoria, se favorezca el diálogo sin juzgar y se contribuya a la generación de contenidos propios de la asignatura; e) tener la participación como elemento transversal durante toda la asignatura, sin penalizar, sin imponer, pero sí como un aspecto integral del que todas las personas se sientan parte; f) generar estructuras de confianza en el aula con pequeños grupos, figuras de liderazgo (rotativas), vías de comunicación diversas, sistema de resolución de conflictos y dinámicas que afiancen la confianza del grupo como parte de los contenidos de la asignatura; g) impulsar actividades más allá del aula y las preguntas en alto que favorezcan una participación libre, como pueden ser visitas externas, talleres complementarios, exposiciones por grupos, debates, seminarios en pequeños grupos, entre otras; $y \mathrm{~h}$ ) hacer visibles las desigualdades de género en la participación. Por parte del profesorado, dirigir la participación si se diera el caso de dicha desigualdad generando procesos de reflexión en torno a estas realidades, haciendo evidente o redistribuyendo los espacios y tiempos de la participación individual. Dando protagonismo, a su vez, a aquellas voces silenciadas o de participación más pasiva. Y, por parte del estudiantado, proponerse la autorregulación en la participación y tomar la palabra como acto de transformación de las desigualdades en el entorno del aula.

En definitiva, concluimos que estas prácticas docentes favorecen una metodología más inclusiva y participativa en las aulas universitarias, lo cual demanda una mayor formación del profesorado en estrategias basadas en igualdad de género. Esta formación queda sujeta, por un lado, al propio interés del docente en evaluar, cuestionar y replantear sus propias prácticas didácticas y, por otro lado, al compromiso de las entidades de 
formación permanente del profesorado en otorgar a las metodologías participativas desde una perspectiva de género un lugar central para el avance hacia docencias transformadoras y generadoras de ciudadanías críticas. Lograr los cambios propuestos pasan por un camino complejo y multidisciplinar. Primeramente, se debe tomar conciencia crítica de nuestras propias prácticas docentes en el aula para después replantear y debatir sobre ellas dando voz y protagonismos al estudiantado. Debemos tener la capacidad de escuchar de manera activa, de promover cambios en nuestras practicas desde un sentimiento de aprender a aprender permanente (Cabello, 2002). En esta línea se ha propuesto una segunda parte del proyecto de innovación aquí presentado, así como la posibilidad de realizar unas jornadas de autoformación del profesorado a partir de los análisis propuestos, ya que, reiteramos, a participar se aprende participando.

\section{REFERENCIAS BIBLIOGRÁFICAS}

Álvarez, J. M. (2001). Evaluar para conocer, examinar para excluir. Madrid: Morata. Barba-Martín, R. (2019). La investigación-acción participativa desde la mirada de las maestras participantes en un proceso de formación permanente del profesorado sobre educación inclusiva (Tesis doctoral inédita). Segovia: Universidad de Valladolid.

Barba-Martín, R.; Martín, Gonzalo \& Barba-Martín, J. J. (2015). «Nuevas perspectivas para el aprendizaje cooperativo desde la investigación-acción: El trabajo colaborativo del claustro y la formación docente». La Peonza. Revista de Educación Física para la paz 10, 85-95.

Barba-Martín, R.; Barba-Martín, J. J. \& Martínez, S. (2016). «La formación continua colaborativa a través de la investigación-acción. Una forma de cambiar las prácticas de aula». Contextos educativos. Revista de Educación, 19, 161-175. http://dx.doi. org/10.18172/con.2769

Barba-Martín, R. \& Sanz, C. (2019). «El proceso de transformación hacia un boletín de evaluación cualitativo e individual en educación infantil mediante investigación-acción participativa». Infancia, Educación y Aprendizaje, 5(2), 504-509. DOI: https:// doi.org/10.22370/ieya.2019.5.2.1728

Berge, B.-M. \& Ve, H. (2000). Action research for gender equity. Buckingham: Open University Press.

Breckmans, I.; Losantos, M.; Loots, G. (2016). «Breaking silence: Exploring motherhood and social transformation in a participatory action research with Alteñan mo- 
thers». Gender, place and cultura, 23(7), 1017-1032, doi: 10.1080/0966369X.2015. 1073702

Caballero, J.; Martín, P. y Villasante, T. (2019). «Debatiendo las metodologías participativas: Un proceso en ocho saltos». EMPIRIA. Revista de Metodología de Ciencias Sociales, 44 septiembre-diciembre, 21-45. DOI/ empiria.43.2019.25350

Cabello, M. J. (2002). Educación permanente y educación social. Málaga: Aljibe.

Colectivo Ioé (2010). «¿Para qué sirve el grupo de discusión? Una revisión crítica del uso de técnicas grupales en los estudios sobre migraciones». Empiria Revista de Metodología de Ciencias Sociales, 19, enero-junio. 73-99.

Crenshaw, K. (2000). «Gender-related aspects of race discrimination». Gender and Racial Discrimination, Noviembre, 21-24.

Fals Borda, O. (1990). El problema de cómo investigar la realidad para transformarla por la praxis. México, Tercer Mundo Editores.

Foote, W. (1991). Participatory action research. Newbury Park: Sage.

Freire, P. (1969). Pedagogía como práctica de libertad. España: S.XX1

Giroux, H. (2019). La guerra del neoliberalismo contra la educación superior. Barcelona: Herder.

Habermas, J. (2000). Teorías y praxis. Madrid: Tecnos.

Hipólito, N.; Martínez, I.; Fernández, S. (2019). Participación social de las mujeres. Madrid: Síntesis.

Ibáñez, J. (1990). «Nuevos avances en la investigación social. La investigación social de segundo orden», Suplemento de Anthropos, 22.

Kemmis, S., y McTaggart, R. (1992). Cómo planificar la investigación acción. Barcelona: Laertes.

Lagarde, M. (2000). Claves feministas para liderazgos entrañables. Managua: puntos de encuentro.

- (2014). El feminismo en mi vida. Hitos, claves y utopias. Madrid: horas y horas.

Martínez Martín, I.. (2016). «Construcción de una pedagogía feminista para una ciudadanía transformadora y contra-hegemónica». Foro de Educación, 14(20), 129-151. doi: 10.14516/fde.2016.014.020.008

- (2017). «Aportes teóricos de la perspectiva de género en la mejora de la educación de las niñas en África». Educación XX1, 20(1), 163-181, doi: 10.5944/educXX1.14474.

- (2018). «Pedagogías Feministas: estrategias para una educación emancipadora y decolonial». Momento-Diálogos em Educação, 27. 3. 350-365

Martínez, L. y Escapa, R. (2008). Guía de formación para la participación social y política de las mujeres. Madrid: Fundación Mujeres. 
McLaren, P. (1997). Pedagogía crítica y cultura depredadora: políticas de oposición en la era postmoderna. Barcelona: Icaria.

Naples, N. (2003). Feminism and method: Etnography, discourse analysis, and activist research. Nueva York: Routledge.

Noffke, S. \& Somekh, B. (2009). The sage handbook of educational action research. Thousand Oaks: SAGE Publications.

Oliveira, V. \& Waldenez, M. (2010). «Trayectorias de investigación acción: Concepciones, objetivos y planteamientos». Revista iberoamericana de Educación 53(5), $1-13$.

Orefice, P. (2014). Ciudadanía emancipada a través de la Investigación Acción Participativa en la búsqueda de nuevos paradigmas de Desarrollo Humano. Santiago de Chile - Florencia: Editorial Universidad Santiago de Chile - Firenze University Press.

Sánchez, M. (2019). Pedagogías queer, Madrid, La Catarata.

Subirats, M. (2017). Coeducación. Apuesta por la libertad. Barcelona: Octaedro.

Tonucci, F. (2009). «Se puede enseñar la participación. Se puede enseñar la democracia». En Revista Investigación en la Escuela 68, 1-14.

Villasante, T., Garrido, F. J. y otros (2002). Metodologías y presupuestos participativos. Madrid, IEPALA y CIMAS. 


\section{ANEXO 1. \\ TABLA DEL PROCESO DE INVESTIGACIÓN-ACCIÓN PARTICIPATIVA APLICADA AL PROYECTO DE INNOVACIÓN DOCENTE}

\begin{tabular}{|c|c|c|}
\hline Temporalización & Tarea & Resultado \\
\hline $\begin{array}{l}\text { Primer mes de la } \\
\text { asignatura }\end{array}$ & $\begin{array}{l}\text { Consensuar indicadores de } \\
\text { observación } \\
\text { Observación participante del } \\
\text { grupo-clase en dos sesiones } \\
\text { aleatorias }\end{array}$ & $\begin{array}{l}\text { Hoja de registro de las } \\
\text { interacciones sociales desde una } \\
\text { perspectiva de género }\end{array}$ \\
\hline $\begin{array}{l}\text { Segundo mes de } \\
\text { la asignatura }\end{array}$ & $\begin{array}{l}\text { Autoanálisis de la participación } \\
\text { individual de cada estudiante } \\
\text { Sesión-taller y grupo de discusión } \\
\text { en torno a la participación propia, } \\
\text { grupal y del profesorado }\end{array}$ & $\begin{array}{l}\text { Análisis de participación } \\
\text { individual } \\
\text { Dinámica «flor de loto». } \\
\text { Reflexiones del grupo de } \\
\text { discusión: ¿qué componente de } \\
\text { género en la construcción de } \\
\text { masculinidades y feminidades } \\
\text { intervienen en la participación?, } \\
\text { ¿cambian las interacciones dentro } \\
\text { del aula si mejoran las dinámicas } \\
\text { de participación en función del } \\
\text { género?, ¿qué favorece o dificulta } \\
\text { la participación? }\end{array}$ \\
\hline $\begin{array}{l}\text { Tercer o cuarto } \\
\text { mes de la } \\
\text { asignatura }\end{array}$ & $\begin{array}{l}\text { Observación participante del } \\
\text { grupo-clase en dos sesiones } \\
\text { aleatorias } \\
\text { Autoanálisis del profesorado de su } \\
\text { percepción de la participación del } \\
\text { grupo clase y su rol en incentivar } \\
\text { la misma }\end{array}$ & $\begin{array}{l}\text { Hoja de registro de las } \\
\text { interacciones sociales desde una } \\
\text { perspectiva de género }\end{array}$ \\
\hline $\begin{array}{l}\text { Final del curso } \\
\text { académico }\end{array}$ & $\begin{array}{l}\text { Jornada formativa para el } \\
\text { profesorado y estudiantes } \\
\text { Encuentro interfacultativo } \\
\text { Propuestas para mejorar la } \\
\text { participación en el aula } \\
\text { Transferencia entre proyectos de } \\
\text { innovación de temática similar }\end{array}$ & $\begin{array}{l}\text { Jornada académica en formato } \\
\text { «coffee-table» } \\
\text { Difusión de los resultados } \\
\text { mediante infografías o } \\
\text { participación en eventos } \\
\text { académicos (congresos, jornadas, } \\
\text { seminarios, etc.) }\end{array}$ \\
\hline
\end{tabular}

Fuente: Elaboración propia. 
ANEXO 2.

HOJA DE REGISTRO CUALITATIVA DE OBSERVACIONES

\begin{tabular}{l|l|l}
\hline \multicolumn{1}{c}{ Ítem a observar } & \multicolumn{1}{c}{$\begin{array}{c}\text { Comentarios } \\
\text { cualitativos }\end{array}$} & Observaciones \\
\hline Nivel de participación general & & \\
\hline ¿Depende la participación general & \\
ítem concreto? & & \\
\hline Tipo de participación & & \\
\hline En los debates generales la participación es... & & \\
\hline
\end{tabular}

Participación por género

\begin{tabular}{l|l|l}
\hline ¿Quién participa más? & & \\
\hline ¿Hay algún perfil que acapara la participación? & & \\
\hline ¿Quién asume las tareas de liderazgo? & & \\
\hline $\begin{array}{l}\text { ¿Quién y cómo se resuelven los conflictos de } \\
\text { participación? }\end{array}$ & & \\
\hline
\end{tabular}

Fuente: Elaboración propia. 
ANEXO 3.

HOJA DE REGISTRO CUALITATIVA DE AUTOANÁLISIS DEL ALUMNADO

\section{AUTOEVALUACIÓN ESTUDIANTES}

¿Soy chica, chico, otros?

¿Soy una persona participativa en clase?

¿Qué considero ser participativa en clase?

¿Lo cumplo?

¿Qué me impide participar?

¿Por qué no participo?

¿Qué favorece mi participación?

- Grupos pequeños

- Dinámicas diversas y cooperativas

- Temas de interés

¿Asumo el liderazgo en alguna ocasión?

- Especificar sí, no y cuándo

¿Consideras si hay diferencias de género en la participación de tu clase?

¿Por qué? Pon algún ejemplo

Fuente: Elaboración propia. 
ANEXO 4.

HOJA DE REGISTRO CUALITATIVA DE AUTOANÁLISIS DEL PROFESORADO

\section{AUTOEVALUACIÓN PROFESORADO}

¿Qué hago como docente para mejorar o impulsar la participación?

- No intervengo, dejo libre la participación

- Introduzco dinámicas variadas

- Corto las intervenciones que acaparan las palabras

- No doy espacios de participación libre

¿Qué favorece la participación inclusiva y en equidad en tu clase?

- Dinámicas cooperativas

- Debates libres

- Mezcla de chicos/as

- Reparto de palabras

- Otros

ANÉCDOTAS LIBRES Y DESTACABLES:

Fuente: Elaboración propia. 